\title{
Drug and Opioid-Involved Overdose Deaths — United States, 2013-2017
}

\author{
Lawrence Scholl, $\mathrm{PhD}^{1}$; Puja Seth, $\mathrm{PhD}^{1}$; Mbabazi Kariisa, $\mathrm{PhD}^{1}$; Nana Wilson, $\mathrm{PhD}^{1}$; Grant Baldwin, $\mathrm{PhD}^{1}$
}

\begin{abstract}
On December 21, 2018, this report was posted as an MMWR Early Release on the MMWR website (https://www.cdc.gov/mmwr).
\end{abstract}

The 63,632 drug overdose deaths in the United States in 2016 represented a $21.4 \%$ increase from 2015; two thirds of these deaths involved an opioid (1). From 2015 to 2016, drug overdose deaths increased in all drug categories examined; the largest increase occurred among deaths involving synthetic opioids other than methadone (synthetic opioids), which includes illicitly manufactured fentanyl (IMF) (1). Since 2013, driven largely by IMF, including fentanyl analogs $(2-4)$, the current wave of the opioid overdose epidemic has been marked by increases in deaths involving synthetic opioids. IMF has contributed to increases in overdose deaths, with geographic differences reported (1). CDC examined state-level changes in death rates involving all drug overdoses in 50 states and the District of Columbia (DC) and those involving synthetic opioids in 20 states, during 2013-2017. In addition, changes in death rates from 2016 to 2017 involving all opioids and opioid subcategories, ${ }^{*}$ were examined by demographics, county urbanization levels, and by 34 states and DC. Among 70,237 drug overdose deaths in 2017, 47,600 (67.8\%) involved an opioid. ${ }^{\dagger}$ From 2013 to 2017 , drug overdose death rates increased in 35 of 50 states and DC, and significant increases in death rates involving synthetic opioids occurred in 15 of 20 states, likely driven by $\operatorname{IMF}(2,3)$. From 2016 to 2017 , overdose deaths involving all opioids and synthetic opioids increased, but deaths involving prescription opioids and heroin remained stable. The opioid overdose epidemic continues to worsen and evolve because of the continuing increase in deaths involving synthetic opioids. Provisional data from 2018 indicate potential improvements in some drug overdose indicators; ${ }^{\circledR}$ however, analysis of final data from 2018 is necessary for confirmation. More timely and comprehensive surveillance data are essential to inform efforts to prevent and respond to opioid overdoses; intensified prevention and response measures are urgently needed to curb deaths involving prescription and illicit opioids, specifically IMF.

Drug overdose deaths were identified in the National Vital Statistics System multiple cause-of-death mortality files, with death certificate data coded using the International Classification of

\footnotetext{
* Natural opioids include morphine and codeine, and semisynthetic opioids include drugs such as oxycodone, hydrocodone, hydromorphone, and oxymorphone. Methadone is a synthetic opioid. Prescription opioids include methadone, natural, and semisynthetic opioids. Synthetic opioids, other than methadone, include drugs such as tramadol and fentanyl. Heroin is an illicit opioid synthesized from morphine that can be a white or brown powder or a black, sticky substance.

${ }^{\dagger}$ https://www.cdc.gov/nchs/products/databriefs/db329.htm.

${ }^{\$}$ https://www.cdc.gov/nchs/nvss/vsrr/drug-overdose-data.htm.

https://www.cdc.gov/nchs/nvss/mortality_public_use_data.htm.
}

Diseases, Tenth Revision (ICD-10) codes X40-44 (unintentional), X60-64 (suicide), X85 (homicide), or Y10-Y14 (undetermined intent). Among deaths with drug overdose as the underlying cause, the type of drug or drug category is indicated by the following ICD-10 multiple cause-of-death codes: opioids (T40.0, T40.1, T40.2, T40.3, T40.4, or T40.6)**; natural/semisynthetic opioids (T40.2); methadone (T40.3); heroin (T40.1); synthetic opioids other than methadone (T40.4); cocaine (T40.5); and psychostimulants with abuse potential (T43.6). ${ }^{\dagger \dagger}$ Some deaths involved more than one type of drug, and these were included in rates for each drug category; thus, categories are not mutually exclusive. ${ }^{\$ \$}$

Annual percent change with statistically significant trends in ageadjusted drug overdose death rates $\$ 9$ for all 50 states and DC from 2013 to 2017 and in age-adjusted death rates involving synthetic opioids for 20 states that met drug specificity criteria*** were analyzed using Joinpoint regression. ${ }^{\dagger \dagger}$ Age-adjusted overdose death rates were examined from 2016 to 2017 for all opioids, prescription opioids (5), heroin, and synthetic opioids. Death rates were stratified by age, sex, racial/ethnic group, urbanization level, ${ }^{\mathbb{S} \$}$ and state. State-level analyses included DC and 34 states with adequate drug

** T40.0 (opium) and T40.6 (other and unspecified narcotics).

†† Psychostimulants with abuse potential include drugs such as methamphetamine and 3,4-methylenedioxy-methamphetamine (MDMA).

$\$ \$$ For example, a death involving both a synthetic opioid other than methadone and heroin would be included in both the synthetic opioid other than methadone and heroin death rates.

99 Age-adjusted death rates were calculated by applying age-specific death rates to the 2000 U.S. Census standard population age distribution https:// www.cdc.gov/nchs/data/nvsr/nvsr61/nvsr61_04.pdf.

*** State-level analyses for synthetic opioid-involved overdose deaths, comparing death rates from 2013 to 2017, included 20 states that met the following criteria: 1) $>80 \%$ of drug overdose death certificates named at least one specific drug in 2013-2017; 2) change from 2013 to 2017 in the percentage of death certificates reporting at least one specific drug was $<10$ percentage points; and 3) $\geq 20$ deaths involving synthetic opioids other than methadone occurred during 2013 and 2017. States whose reporting of any specific drug or drugs involved in an overdose changed by $\geq 10$ percentage points from 2013 to 2017 were excluded because drugspecific overdose numbers and rates might have changed substantially from 2013 to 2017 as a result of changes in reporting.

扩 For all analyses, a p-value of $<0.05$ was considered to be statistically significant. https://surveillance.cancer.gov/joinpoint/.

$\$ \$ \$ S$ Categories of 2013 NCHS Urban-Rural Classification Scheme for Counties (https://www.cdc.gov/nchs/data_access/urban_rural.htm). Large central metro: Counties in metropolitan statistical areas (MSAs) of $\geq 1$ million population that 1) contain the entire population of largest principal city of the MSA, or 2) have their entire population contained in the largest principal city of the MSA, or 3) contain at least 250,000 inhabitants of any principal city of the MSA; Large fringe metro: Counties in MSAs of $\geq 1$ million population that did not qualify as large central metro counties; Medium metro: Counties in MSAs of populations of 250,000-999,999; Small metro: Counties in MSAs of populations <250,000; Micropolitan (nonmetropolitan counties): counties in micropolitan statistical areas; Noncore (nonmetropolitan counties): nonmetropolitan counties that did not qualify as micropolitan. 
specificity data for 2016 and 2017.999 Analyses comparing changes in death rates from 2016 to 2017 used z-tests when the number of deaths was $\geq 100$ and nonoverlapping confidence intervals based on a gamma distribution when the number was $<100$.*** $^{* * *}$

Drug overdoses resulted in 70,237 deaths during 2017; among these, 47,600 (67.8\%) involved opioids (14.9 per 100,000 population), representing a $12.0 \%$ rate increase from 2016 (Table 1 ).

\footnotetext{
999 State-level analyses comparing death rates from 2016 to 2017 included 34 states and DC that met the following criteria: 1) $>80 \%$ of drug overdose death certificates named at least one specific drug in 2016 and 2017; 2) change from 2016 to 2017 in the percentage of death certificates reporting at least one specific drug was $<10$ percentage points; and 3) $\geq 20$ deaths occurred during 2016 and 2017 in at least two opioid subcategories examined. States whose reporting of any specific drug or drugs involved in an overdose changed by $\geq 10$ percentage points from 2016 to 2017 were excluded because drug-specific overdose numbers and rates might have changed substantially from 2016 to 2017 as a result of changes in reporting.

**** Z-tests were used if the number of deaths was $\geq 100$, and a $\mathrm{p}$-value of $<0.05$ was considered to be statistically significant. Nonoverlapping confidence intervals based on the gamma method were used if the number of deaths was $<100$ in 2015 or 2016 . Note that the method of comparing confidence intervals is a conservative method for statistical significance; caution should be observed when interpreting a nonsignificant difference when the lower and upper limits being compared overlap only slightly.
}

Synthetic opioids were involved in $59.8 \%$ of all opioid-involved overdose deaths; the rate increased by $45.2 \%$ from 2016 to 2017 (Table 2). From 2013 through 2017, overdose death rates increased significantly in 35 states and DC; 15 of 20 states that met drug specificity criteria had significant increases in overdose death rates involving synthetic opioids (Figure). From 2016 to 2017, death rates involving cocaine and psychostimulants increased $34.4 \%$ (from 3.2 to 4.3 per 100,000 ) and $33.3 \%$ (from 2.4 to 3.2 per 100,000), respectively, likely contributing to increases in drug overdose deaths; however, rates remained stable for deaths involving prescription opioids ( 5.2 per 100,000$)$ (Table 1) and heroin (4.9) (Table 2).

From 2016 to 2017, opioid-involved overdose deaths increased among males and females and among persons aged $\geq 25$ years, non-Hispanic whites (whites), non-Hispanic blacks (blacks), and Hispanics (Table 1). The largest relative change occurred among blacks (25.2\%), and the largest absolute rate increase was among males aged 25-44 years (an increase of 4.6 per 100,000). The largest relative change among age groups was for persons aged $\geq 65$ years $(17.2 \%)$. Counties in medium metro

TABLE 1. Annual number and age-adjusted rate of drug overdose deaths* involving all opioids ${ }^{\dagger}$ and prescription opioids, ${ }^{\S, \uparrow}$ by sex, age, race and Hispanic origin, ${ }^{* *}$ urbanization level, ${ }^{\dagger \dagger}$ and selected states ${ }^{\S \S}$ — United States, 2016 and 2017

\begin{tabular}{|c|c|c|c|c|c|c|c|c|c|c|c|c|}
\hline \multirow[b]{3}{*}{ Decedent characteristic } & \multicolumn{6}{|c|}{ All opioids } & \multicolumn{6}{|c|}{ Prescription opioids } \\
\hline & \multicolumn{2}{|c|}{2016} & \multicolumn{2}{|c|}{2017} & \multicolumn{2}{|c|}{$\begin{array}{l}\text { Change from } \\
2016 \text { to } 2017^{\text {ๆ }}\end{array}$} & \multicolumn{2}{|c|}{2016} & \multicolumn{2}{|c|}{2017} & \multicolumn{2}{|c|}{$\begin{array}{l}\text { Change from } \\
2016 \text { to } 2017^{\text {ๆๆ }}\end{array}$} \\
\hline & No. & Rate & No. & Rate & $\begin{array}{l}\text { Absolute } \\
\text { rate change }\end{array}$ & $\begin{array}{l}\% \text { Change } \\
\text { in rate }\end{array}$ & No. & Rate & No. & Rate & $\begin{array}{l}\text { Absolute } \\
\text { rate change }\end{array}$ & $\begin{array}{l}\% \text { Change } \\
\text { in rate }\end{array}$ \\
\hline All & 42,249 & 13.3 & 47,600 & 14.9 & $1.6^{* * *}$ & $12.0 * * *$ & 17,087 & 5.2 & 17,029 & 5.2 & 0.0 & 0.0 \\
\hline \multicolumn{13}{|l|}{ Sex } \\
\hline Male & 28,498 & 18.1 & 32,337 & 20.4 & $2.3^{* * *}$ & $12.7^{* * *}$ & 9,978 & 6.2 & 9,873 & 6.1 & -0.1 & -1.6 \\
\hline Female & 13,751 & 8.5 & 15,263 & 9.4 & $0.9^{* * *}$ & $10.6^{* * *}$ & 7,109 & 4.3 & 7,156 & 4.2 & -0.1 & -2.3 \\
\hline \multicolumn{13}{|l|}{ Age group (yrs) } \\
\hline $0-14$ & 83 & 0.1 & 79 & 0.1 & 0.0 & 0.0 & 60 & 0.1 & 50 & 0.1 & 0.0 & 0.0 \\
\hline $15-24$ & 4,027 & 9.3 & 4,094 & 9.5 & 0.2 & 2.2 & 1,146 & 2.6 & 1,050 & 2.4 & -0.2 & -7.7 \\
\hline $25-34$ & 11,552 & 25.9 & 13,181 & 29.1 & $3.2^{* * *}$ & $12.4^{* * *}$ & 3,442 & 7.7 & 3,408 & 7.5 & -0.2 & -2.6 \\
\hline $35-44$ & 9,747 & 24.1 & 11,149 & 27.3 & $3.2^{* * * *}$ & $13.3^{* * *}$ & 3,727 & 9.2 & 3,714 & 9.1 & -0.1 & -1.1 \\
\hline $45-54$ & 9,074 & 21.2 & 10,207 & 24.1 & $2.9^{* * *}$ & $13.7^{* * *}$ & 4,307 & 10.1 & 4,238 & 10.0 & -0.1 & -1.0 \\
\hline $55-64$ & 6,321 & 15.2 & 7,153 & 17.0 & $1.8^{* * *}$ & $11.8^{* * *}$ & 3,489 & 8.4 & 3,509 & 8.4 & 0.0 & 0.0 \\
\hline$\geq 65$ & 1,441 & 2.9 & 1,724 & 3.4 & $0.5^{* * *}$ & $17.2^{* * *}$ & 915 & 1.9 & 1,055 & 2.1 & $0.2^{* * *}$ & $10.5^{* * *}$ \\
\hline \multicolumn{13}{|l|}{ Sex and age group (yrs) } \\
\hline Male 15-24 & 2,986 & 13.4 & 2,885 & 13.0 & -0.4 & -3.0 & 852 & 3.8 & 728 & 3.3 & $-0.5^{* * *}$ & $-13.2^{* * *}$ \\
\hline Male 25-44 & 15,137 & 35.4 & 17,352 & 40.0 & $4.6^{* * *}$ & $13.0^{* * *}$ & 4,527 & 10.6 & 4,516 & 10.4 & -0.2 & -1.9 \\
\hline Male 45-64 & 9,519 & 23.2 & 11,061 & 26.9 & $3.7^{* * *}$ & $15.9^{* * *}$ & 4,124 & 10.0 & 4,089 & 9.9 & -0.1 & -1.0 \\
\hline Female 15-24 & 1,041 & 4.9 & 1,209 & 5.7 & $0.8^{* * *}$ & $16.3^{* * *}$ & 294 & 1.4 & 322 & 1.5 & 0.1 & 7.1 \\
\hline Female 25-44 & 6,162 & 14.5 & 6,978 & 16.3 & $1.8^{* * * *}$ & $12.4^{* * *}$ & 2,642 & 6.2 & 2,606 & 6.1 & -0.1 & -1.6 \\
\hline Female 45-64 & 5,876 & 13.6 & 6,299 & 14.6 & $1.0^{* * * *}$ & $7.4^{* * *}$ & 3,672 & 8.5 & 3,658 & 8.5 & 0.0 & 0.0 \\
\hline \multicolumn{13}{|l|}{ Race and Hispanic origin** } \\
\hline White, non-Hispanic & 33,450 & 17.5 & 37,113 & 19.4 & $1.9 * * *$ & $10.9^{* * *}$ & 14,167 & 7.0 & 13,900 & 6.9 & -0.1 & -1.4 \\
\hline Black, non-Hispanic & 4,374 & 10.3 & 5,513 & 12.9 & $2.6^{* * *}$ & $25.2^{* * *}$ & 1,392 & 3.3 & 1,508 & 3.5 & 0.2 & 6.1 \\
\hline Hispanic & 3,440 & 6.1 & 3,932 & 6.8 & $0.7^{* * *}$ & $11.5^{* * *}$ & 1,133 & 2.1 & 1,211 & 2.2 & 0.1 & 4.8 \\
\hline $\begin{array}{l}\text { American Indian/Alaska } \\
\text { Native, non-Hispanic }\end{array}$ & 369 & 13.9 & 408 & 15.7 & 1.8 & 12.9 & 173 & 6.5 & 187 & 7.2 & 0.7 & 10.8 \\
\hline $\begin{array}{l}\text { Asian/Pacific Islander, } \\
\text { non-Hispanic }\end{array}$ & 323 & 1.5 & 348 & 1.6 & 0.1 & 6.7 & 131 & 0.7 & 130 & 0.6 & -0.1 & -14.3 \\
\hline \multicolumn{13}{|c|}{ County urbanization level ${ }^{\dagger \dagger}$} \\
\hline Large central metro & 12,903 & 12.5 & 14,518 & 13.9 & $1.4^{* * *}$ & $11.2^{* * *}$ & 4,930 & 4.7 & 4,945 & 4.7 & 0.0 & 0.0 \\
\hline Large fringe metro & 11,993 & 15.4 & 13,594 & 17.2 & $1.8^{* * *}$ & $11.7^{* * *}$ & 4,209 & 5.2 & 4,273 & 5.2 & 0.0 & 0.0 \\
\hline Medium metro & 9,264 & 14.3 & 10,561 & 16.2 & $1.9^{* * *}$ & $13.3^{* * *}$ & 3,988 & 6.0 & 3,951 & 5.9 & -0.1 & -1.7 \\
\hline Small metro & 3,224 & 11.7 & 3,560 & 12.9 & $1.2^{* * *}$ & $10.3^{* * *}$ & 1,471 & 5.2 & 1,479 & 5.2 & 0.0 & 0.0 \\
\hline
\end{tabular}

See table footnotes on next page. 
TABLE 1. (Continued) Annual number and age-adjusted rate of drug overdose deaths* involving all opioids ${ }^{\dagger}$ and prescription opioids, ${ }^{\S, \uparrow}$ by sex, age, race and Hispanic origin, ${ }^{* *}$ urbanization level, $^{+\dagger}$ and selected states ${ }^{\S \S}$ — United States, 2016 and 2017

\begin{tabular}{|c|c|c|c|c|c|c|c|c|c|c|c|c|}
\hline \multirow[b]{3}{*}{ Decedent characteristic } & \multicolumn{6}{|c|}{ All opioids } & \multicolumn{6}{|c|}{ Prescription opioids } \\
\hline & \multicolumn{2}{|c|}{2016} & \multicolumn{2}{|c|}{2017} & \multicolumn{2}{|c|}{$\begin{array}{l}\text { Change from } \\
2016 \text { to } 2017^{\text {ศึ }}\end{array}$} & \multicolumn{2}{|c|}{2016} & \multicolumn{2}{|c|}{2017} & \multicolumn{2}{|c|}{$\begin{array}{l}\text { Change from } \\
2016 \text { to } 2017^{\text {१ी }}\end{array}$} \\
\hline & No. & Rate & No. & Rate & $\begin{array}{l}\text { Absolute } \\
\text { rate change }\end{array}$ & $\begin{array}{l}\% \text { Change } \\
\text { in rate }\end{array}$ & No. & Rate & No. & Rate & $\begin{array}{l}\text { Absolute } \\
\text { rate change }\end{array}$ & $\begin{array}{l}\% \text { Change } \\
\text { in rate }\end{array}$ \\
\hline Micropolitan (nonmetro) & 3,068 & 12.1 & 3,462 & 13.9 & $1.8^{* * *}$ & $14.9^{* * *}$ & 1,475 & 5.7 & 1,440 & 5.6 & -0.1 & -1.8 \\
\hline Noncore (nonmetro) & 1,797 & 10.5 & 1,905 & 11.2 & 0.7 & 6.7 & 1,014 & 5.7 & 941 & 5.3 & -0.4 & -7.0 \\
\hline \multirow{2}{*}{\multicolumn{13}{|c|}{$\begin{array}{l}\text { Selected states } \S \S \\
\text { States with very good to excellent reporting }(n=27)\end{array}$}} \\
\hline & & & & & & & & & & & & \\
\hline Alaska & 94 & 12.5 & 102 & 13.9 & 1.4 & 11.2 & 51 & 6.8 & 51 & 7.0 & 0.2 & 2.9 \\
\hline Connecticut & 855 & 24.5 & 955 & 27.7 & $3.2^{* * *}$ & $13.1^{* * *}$ & 264 & 7.2 & 273 & 7.7 & 0.5 & 6.9 \\
\hline District of Columbia & 209 & 30.0 & 244 & 34.7 & 4.7 & 15.7 & 66 & 9.3 & 58 & 8.4 & -0.9 & -9.7 \\
\hline Georgia & 918 & 8.8 & 1,014 & 9.7 & $0.9^{* * *}$ & $10.2^{* * *}$ & 536 & 5.1 & 568 & 5.4 & 0.3 & 5.9 \\
\hline Hawaii & 77 & 5.2 & 53 & 3.4 & -1.8 & -34.6 & 55 & 3.6 & 40 & 2.5 & -1.1 & -30.6 \\
\hline Illinois & 1,947 & 15.3 & 2,202 & 17.2 & $1.9^{* * *}$ & $12.4 * * *$ & 479 & 3.7 & 623 & 4.8 & $1.1^{* * *}$ & $29.7^{* * *}$ \\
\hline lowa & 183 & 6.2 & 206 & 6.9 & 0.7 & 11.3 & 92 & 3.1 & 104 & 3.4 & 0.3 & 9.7 \\
\hline Maine & 301 & 25.2 & 360 & 29.9 & $4.7^{* * *}$ & $18.7^{* * *}$ & 154 & 12.5 & 100 & 7.6 & $-4.9^{* * *}$ & $-39.2^{* * *}$ \\
\hline Maryland & 1,821 & 29.7 & 1,985 & 32.2 & $2.5^{* * *}$ & $8.4^{* * *}$ & 812 & 13.1 & 711 & 11.5 & $-1.6^{* * *}$ & $-12.2^{* * *}$ \\
\hline Massachusetts & 1,990 & 29.7 & 1,913 & 28.2 & -1.5 & -5.1 & 351 & 4.9 & 321 & 4.6 & -0.3 & -6.1 \\
\hline Nevada & 408 & 13.3 & 412 & 13.3 & 0.0 & 0.0 & 275 & 8.9 & 276 & 8.7 & -0.2 & -2.2 \\
\hline New Hampshire & 437 & 35.8 & 424 & 34.0 & -1.8 & -5.0 & 89 & 6.5 & 62 & 4.8 & -1.7 & -26.2 \\
\hline New Mexico & 349 & 17.5 & 332 & 16.7 & -0.8 & -4.6 & 186 & 9.2 & 171 & 8.5 & -0.7 & -7.6 \\
\hline New York & 3,009 & 15.1 & 3,224 & 16.1 & $1.0^{* * *}$ & $6.6^{* * *}$ & 1,100 & 5.4 & 1,044 & 5.1 & -0.3 & -5.6 \\
\hline North Carolina & 1,506 & 15.4 & 1,953 & 19.8 & $4.4^{* * *}$ & $28.6^{* * *}$ & 695 & 6.9 & 659 & 6.5 & -0.4 & -5.8 \\
\hline Ohio & 3,613 & 32.9 & 4,293 & 39.2 & $6.3^{* * *}$ & $19.1^{* * *}$ & 867 & 7.7 & 947 & 8.4 & 0.7 & 9.1 \\
\hline Oklahoma & 444 & 11.6 & 388 & 10.2 & -1.4 & -12.1 & 322 & 8.4 & 251 & 6.7 & $-1.7^{* * *}$ & $-20.2^{* * *}$ \\
\hline Oregon & 312 & 7.6 & 344 & 8.1 & 0.5 & 6.6 & 165 & 3.9 & 154 & 3.5 & -0.4 & -10.3 \\
\hline Rhode Island & 279 & 26.7 & 277 & 26.9 & 0.2 & 0.7 & 114 & 10.5 & 99 & 8.8 & -1.7 & -16.2 \\
\hline South Carolina & 628 & 13.1 & 749 & 15.5 & $2.4^{* * *}$ & $18.3^{* * *}$ & 381 & 7.8 & 345 & 7.1 & -0.7 & -9.0 \\
\hline Tennessee & 1,186 & 18.1 & 1,269 & 19.3 & 1.2 & 6.6 & 739 & 11.1 & 644 & 9.6 & $-1.5^{* * *}$ & $-13.5 * * *$ \\
\hline Utah & 466 & 16.4 & 456 & 15.5 & -0.9 & -5.5 & 349 & 12.5 & 315 & 10.8 & -1.7 & -13.6 \\
\hline Vermont & 101 & 18.4 & 114 & 20.0 & 1.6 & 8.7 & 35 & 5.9 & 40 & 6.3 & 0.4 & 6.8 \\
\hline Virginia & 1,130 & 13.5 & 1,241 & 14.8 & $1.3^{* * *}$ & $9.6^{* * *}$ & 400 & 4.7 & 404 & 4.7 & 0.0 & 0.0 \\
\hline Washington & 709 & 9.4 & 742 & 9.6 & 0.2 & 2.1 & 388 & 5.0 & 343 & 4.3 & $-0.7^{* * *}$ & $-14.0 * * *$ \\
\hline West Virginia & 733 & 43.4 & 833 & 49.6 & $6.2^{* * * *}$ & $14.3^{* * *}$ & 340 & 19.7 & 304 & 17.2 & -2.5 & -12.7 \\
\hline Wisconsin & 866 & 15.8 & 926 & 16.9 & 1.1 & 7.0 & 382 & 6.7 & 362 & 6.4 & -0.3 & -4.5 \\
\hline \multicolumn{13}{|c|}{ States with good reporting $(n=8)$} \\
\hline Arizona & 769 & 11.4 & 928 & 13.5 & $2.1^{* * *}$ & $18.4^{* * *}$ & 380 & 5.6 & 414 & 5.9 & 0.3 & 5.4 \\
\hline California & 2,012 & 4.9 & 2,199 & 5.3 & $0.4^{* * *}$ & $8.2^{* * *}$ & 1,172 & 2.8 & 1,169 & 2.8 & 0.0 & 0.0 \\
\hline Colorado & 536 & 9.5 & 578 & 10.0 & 0.5 & 5.3 & 258 & 4.5 & 300 & 5.1 & 0.6 & 13.3 \\
\hline Kentucky & 989 & 23.6 & 1,160 & 27.9 & $4.3^{* * *}$ & $18.2^{* * *}$ & 429 & 10.0 & 433 & 10.2 & 0.2 & 2.0 \\
\hline Michigan & 1,762 & 18.5 & 2,033 & 21.2 & $2.7^{* * *}$ & $14.6^{* * *}$ & 678 & 7.0 & 633 & 6.5 & -0.5 & -7.1 \\
\hline Minnesota & 396 & 7.4 & 422 & 7.8 & 0.4 & 5.4 & 195 & 3.6 & 195 & 3.6 & 0.0 & 0.0 \\
\hline Missouri & 914 & 15.9 & 952 & 16.5 & 0.6 & 3.8 & 268 & 4.5 & 253 & 4.1 & -0.4 & -8.9 \\
\hline Texas & 1,375 & 4.9 & 1,458 & 5.1 & 0.2 & 4.1 & 617 & 2.2 & 646 & 2.3 & 0.1 & 4.5 \\
\hline
\end{tabular}

Source: National Vital Statistics System, Mortality file.

* Deaths are classified using the International Classification of Diseases, Tenth Revision (ICD-10). Drug overdose deaths are identified using underlying cause-of-death codes X40-X44, X60-X64, X85, and Y10-Y14. Rates are age-adjusted using the direct method and the 2000 U.S. standard population, except for age-specific crude rates. All rates are per 100,000 population.

${ }^{\dagger}$ Drug overdose deaths, as defined, that have opium (T40.0), heroin (T40.1), natural and semisynthetic opioids (T40.2), methadone (T40.3), synthetic opioids other than methadone (T40.4), or other and unspecified narcotics (T40.6) as a contributing cause.

$\S$ Drug overdose deaths, as defined, that have natural and semisynthetic opioids (T40.2) or methadone (T40.3) as a contributing cause.

१ Categories of deaths are not exclusive because deaths might involve more than one drug. Summing of categories will result in more than the total number of deaths in a year.

** Data for Hispanic origin should be interpreted with caution; studies comparing Hispanic origin on death certificates and on census surveys have shown inconsistent reporting on Hispanic ethnicity. Potential race misclassification might lead to underestimates for certain categories, primarily American Indian/Alaska Native non-Hispanic and Asian/Pacific Islander non-Hispanic decedents. https://www.cdc.gov/nchs/data/series/sr_02/sr02_172.pdf.

${ }^{+\dagger}$ By 2013 urbanization classification (https://www.cdc.gov/nchs/data_access/urban_rural.htm).

$\S \S$ Analyses were limited to states meeting the following criteria. For states with very good to excellent reporting, $\geq 90 \%$ of drug overdose deaths mention at least one specific drug in 2016, with the change in drug overdose deaths mentioning at least one specific drug differing by $<10$ percentage points from 2016 to 2017 . States with good reporting had $80 \%$ to $<90 \%$ of drug overdose deaths mention at least one specific drug in 2016 , with the change in the percentage of drug overdose deaths mentioning at least one specific drug differing by $<10$ percentage points from 2016 to 2017 . States included also were required to have stable rate estimates, based on $\geq 20$ deaths, in at least two drug categories (i.e., opioids, prescription opioids, synthetic opioids other than methadone, and heroin).

กा Absolute rate change is the difference between 2016 and 2017 rates. Percent change is the absolute rate change divided by the 2016 rate, multiplied by 100. Nonoverlapping confidence intervals based on the gamma method were used if the number of deaths was $<100$ in 2016 or 2017, and z-tests were used if the number of deaths was $\geq 100$ in both 2016 and 2017.

*** Statistically significant (P-value $<0.05)$. 
TABLE 2. Annual number and age-adjusted rate of drug overdose deaths* involving heroin ${ }^{\dagger}$ and synthetic opioids other than methadone, ${ }^{\S, \uparrow}$ by sex, age, race and Hispanic origin, ${ }^{* *}$ urbanization level, ${ }^{\dagger \dagger}$ and selected states ${ }^{\S \S}$ — United States, 2016 and 2017

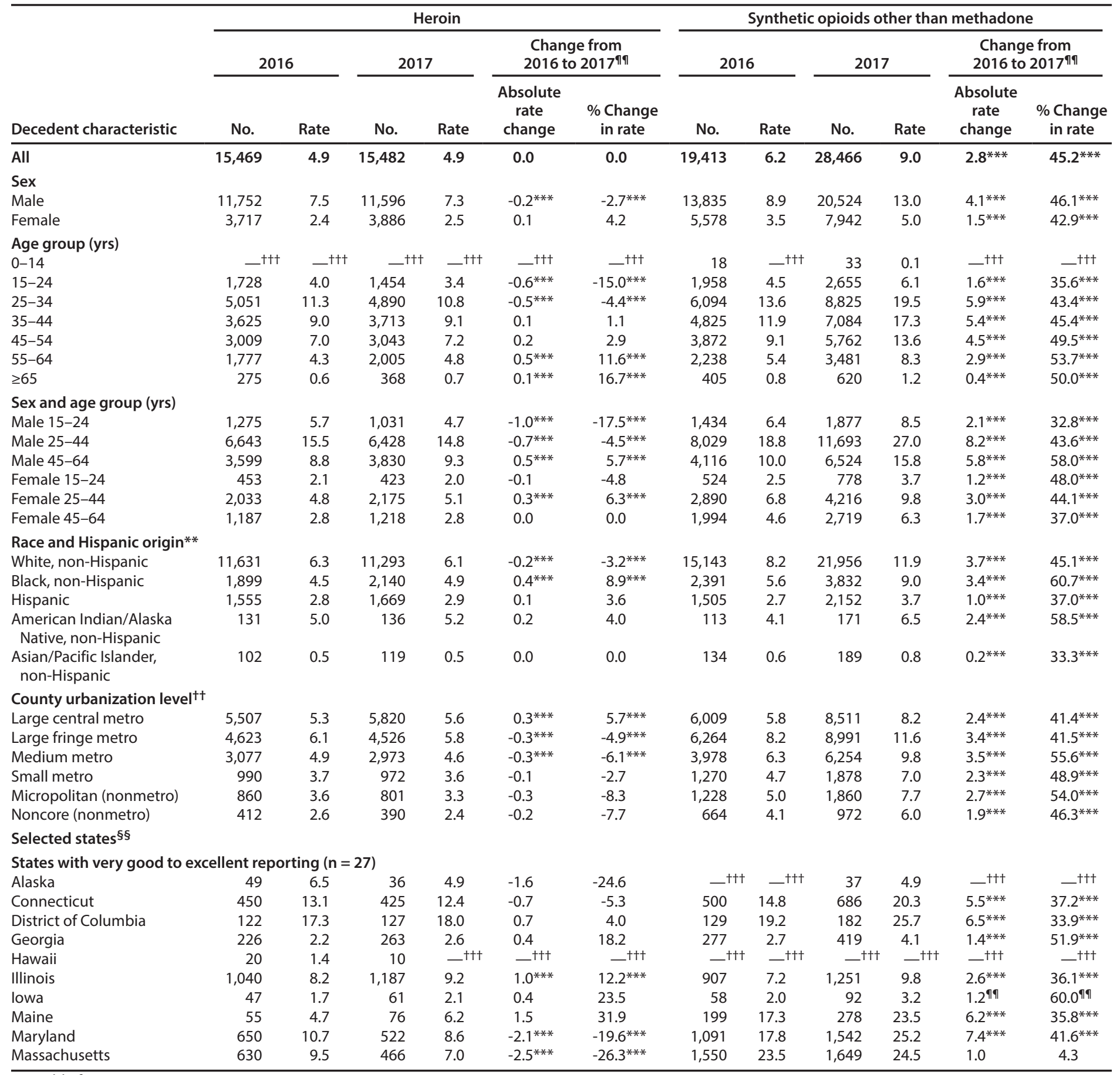

See table footnotes on next page. 
TABLE 2. (Continued) Annual number and age-adjusted rate of drug overdose deaths* involving heroin ${ }^{\dagger}$ and synthetic opioids other than methadone, ${ }^{\S, \pi}$ by sex, age, race and Hispanic origin, ${ }^{* *}$ urbanization level, ${ }^{\dagger \dagger}$ and selected states ${ }^{\S \S}$ — United States, 2016 and 2017

\begin{tabular}{|c|c|c|c|c|c|c|c|c|c|c|c|c|}
\hline \multirow[b]{3}{*}{ Decedent characteristic } & \multicolumn{6}{|c|}{ Heroin } & \multicolumn{6}{|c|}{ Synthetic opioids other than methadone } \\
\hline & \multicolumn{2}{|c|}{2016} & \multicolumn{2}{|c|}{2017} & \multicolumn{2}{|c|}{$\begin{array}{l}\text { Change from } \\
2016 \text { to } 2017^{\text {१ิ }}\end{array}$} & \multicolumn{2}{|c|}{2016} & \multicolumn{2}{|c|}{2017} & \multicolumn{2}{|c|}{$\begin{array}{l}\text { Change from } \\
2016 \text { to } 2017^{\text {ๆी }}\end{array}$} \\
\hline & No. & Rate & No. & Rate & $\begin{array}{l}\text { Absolute } \\
\text { rate } \\
\text { change }\end{array}$ & $\begin{array}{l}\% \text { Change } \\
\text { in rate }\end{array}$ & No. & Rate & No. & Rate & $\begin{array}{l}\text { Absolute } \\
\text { rate } \\
\text { change }\end{array}$ & $\begin{array}{c}\% \text { Change } \\
\text { in rate }\end{array}$ \\
\hline Nevada & 86 & 2.9 & 94 & 3.1 & 0.2 & 6.9 & 53 & 1.7 & 66 & 2.2 & 0.5 & 29.4 \\
\hline New Hampshire & 34 & 2.8 & 28 & 2.4 & -0.4 & -14.3 & 363 & 30.3 & 374 & 30.4 & 0.1 & 0.3 \\
\hline New Mexico & 161 & 8.2 & 144 & 7.4 & -0.8 & -9.8 & 78 & 4.0 & 75 & 3.7 & -0.3 & -7.5 \\
\hline New York & 1,307 & 6.5 & 1,356 & 6.8 & 0.3 & 4.6 & 1,641 & 8.3 & 2,238 & 11.3 & $3.0^{* * *}$ & $36.1^{* * *}$ \\
\hline North Carolina & 544 & 5.7 & 537 & 5.6 & -0.1 & -1.8 & 601 & 6.2 & 1,285 & 13.2 & $7.0^{* * * *}$ & $112.9 * * *$ \\
\hline Ohio & 1,478 & 13.5 & 1,000 & 9.2 & $-4.3^{* * *}$ & $-31.9^{* * *}$ & 2,296 & 21.1 & 3,523 & 32.4 & $11.3^{* * *}$ & $53.6^{* * *}$ \\
\hline Oklahoma & 53 & 1.4 & 61 & 1.6 & 0.2 & 14.3 & 98 & 2.5 & 102 & 2.6 & 0.1 & 4.0 \\
\hline Oregon & 114 & 2.9 & 124 & 3.0 & 0.1 & 3.4 & 43 & 1.1 & 85 & 2.1 & $1.0^{* * *}$ & $90.9^{* * *}$ \\
\hline Rhode Island & 25 & 2.5 & 14 & $-{ }^{t+\dagger}$ & $-{ }^{\mathrm{t \dagger}+}$ & - $^{\dagger+t}$ & 182 & 17.8 & 201 & 20.1 & 2.3 & 12.9 \\
\hline South Carolina & 115 & 2.5 & 153 & 3.2 & 0.7 & 28.0 & 237 & 5.0 & 404 & 8.5 & $3.5^{* * *}$ & $70.0^{* * *}$ \\
\hline Tennessee & 260 & 4.1 & 311 & 4.8 & 0.7 & 17.1 & 395 & 6.2 & 590 & 9.3 & $3.1^{* * *}$ & $50.0^{* * *}$ \\
\hline Utah & 166 & 5.6 & 147 & 4.8 & -0.8 & -14.3 & 72 & 2.5 & 92 & 3.1 & 0.6 & 24.0 \\
\hline Vermont & 45 & 8.7 & 41 & 7.3 & -1.4 & -16.1 & 53 & 10.1 & 77 & 13.8 & 3.7 & 36.6 \\
\hline Virginia & 450 & 5.5 & 556 & 6.7 & $1.2^{* * *}$ & $21.8^{* * *}$ & 648 & 7.9 & 829 & 10.0 & $2.1^{* * * *}$ & $26.6^{* * *}$ \\
\hline Washington & 283 & 3.9 & 306 & 4.0 & 0.1 & 2.6 & 93 & 1.3 & 143 & 1.9 & $0.6^{* * *}$ & $46.2^{* * *}$ \\
\hline West Virginia & 235 & 14.9 & 244 & 14.9 & 0.0 & 0.0 & 435 & 26.3 & 618 & 37.4 & $11.1^{* * *}$ & $42.2^{* * *}$ \\
\hline Wisconsin & 389 & 7.3 & 414 & 7.8 & 0.5 & 6.8 & 288 & 5.3 & 466 & 8.6 & $3.3^{* * *}$ & $62.3^{* * *}$ \\
\hline \multicolumn{13}{|c|}{ States with good reporting $(n=8)$} \\
\hline Arizona & 299 & 4.5 & 334 & 5.0 & 0.5 & 11.1 & 123 & 1.8 & 267 & 4.0 & $2.2^{* * *}$ & $122.2^{* * *}$ \\
\hline California & 587 & 1.4 & 715 & 1.7 & $0.3^{* * *}$ & $21.4^{* * *}$ & 355 & 0.9 & 536 & 1.3 & $0.4^{* * *}$ & $44.4^{* * *}$ \\
\hline Colorado & 234 & 4.2 & 224 & 3.9 & -0.3 & -7.1 & 72 & 1.3 & 112 & 2.0 & $0.7^{* * *}$ & $53.8^{* * *}$ \\
\hline Kentucky & 311 & 7.6 & 269 & 6.6 & -1.0 & -13.2 & 465 & 11.5 & 780 & 19.1 & $7.6^{* * * *}$ & $66.1^{* * *}$ \\
\hline Michigan & 727 & 7.6 & 783 & 8.2 & 0.6 & 7.9 & 921 & 9.8 & 1,368 & 14.4 & $4.6^{* * * *}$ & $46.9^{* * * *}$ \\
\hline Minnesota & 149 & 2.8 & 111 & 2.0 & $-0.8^{* * * *}$ & $-28.6^{* * *}$ & 99 & 1.9 & 184 & 3.5 & $1.6^{* * * *}$ & $84.2^{* * * *}$ \\
\hline Missouri & 380 & 6.7 & 299 & 5.3 & $-1.4^{* * *}$ & $-20.9 * * *$ & 441 & 7.8 & 618 & 10.9 & $3.1^{* * *}$ & $39.7^{* * *}$ \\
\hline Texas & 530 & 1.9 & 569 & 2.0 & 0.1 & 5.3 & 250 & 0.9 & 348 & 1.2 & $0.3^{* * *}$ & $33.3^{* * * *}$ \\
\hline
\end{tabular}

Source: National Vital Statistics System, Mortality file.

* Deaths are classified using the International Classification of Diseases, Tenth Revision (ICD-10). Drug overdose deaths are identified using underlying cause-of-death codes X40-X44, X60-X64, X85, and Y10-Y14. Rates are age-adjusted using the direct method and the 2000 U.S. standard population, except for age-specific crude rates. All rates are per 100,000 population.

† Drug overdose deaths, as defined, that have heroin (T40.1) as a contributing cause.

$\S$ Drug overdose deaths, as defined, that have semisynthetic opioids other than methadone (T40.4) as a contributing cause.

" Categories of deaths are not exclusive as deaths might involve more than one drug. Summing of categories will result in more than the total number of deaths in a year.

** Data on Hispanic origin should be interpreted with caution; studies comparing Hispanic origin on death certificates and on census surveys have shown inconsistent reporting on Hispanic ethnicity. Potential race misclassification might lead to underestimates for certain categories, primarily American Indian/Alaska Native non-Hispanic and Asian/Pacific Islander non-Hispanic decedents. https://www.cdc.gov/nchs/data/series/sr_02/sr02_172.pdf.

${ }^{+\dagger}$ By 2013 urbanization classification (https://www.cdc.gov/nchs/data_access/urban_rural.htm).

$\S \S$ Analyses were limited to states meeting the following criteria. For states with very good to excellent reporting, $\geq 90 \%$ of drug overdose deaths mention at least one specific drug in 2016, with the change in drug overdose deaths mentioning at least one specific drug differing by $<10$ percentage points from 2016 to 2017 . States with good reporting had $80 \%$ to $<90 \%$ of drug overdose deaths mention at least one specific drug in 2016 , with the change in the percentage of drug overdose deaths mentioning at least one specific drug differing by $<10$ percentage points from 2016 to 2017 . States included also were required to have stable rate estimates, based on $\geq 20$ deaths, in at least two drug categories (i.e., opioids, prescription opioids, synthetic opioids other than methadone, and heroin).

Iी Absolute rate change is the difference between 2016 and 2017 rates. Percent change is the absolute rate change divided by the 2016 rate, multiplied by 100. Nonoverlapping confidence intervals based on the gamma method were used if the number of deaths was $<100$ in 2016 or 2017 , and z-tests were used if the number of deaths was $\geq 100$ in both 2016 and 2017. Note that the method of comparing confidence intervals is a conservative method for statistical significance; caution should be observed when interpreting a nonsignificant difference when the lower and upper limits being compared overlap only slightly. Confidence intervals of 2016 and 2017 rates of synthetic opioid-involved deaths in lowa overlapped only slightly: $(1.40,2.39),(2.36,3.59)$.

*** Statistically significant (P-value $<0.05)$.

${ }^{+t+}$ Cells with $\leq 9$ deaths are not reported. Rates based on $<20$ deaths are not considered reliable and are not reported. 
FIGURE. Age-adjusted rates* of drug overdose deaths and deaths involving synthetic opioids other than methadone, ${ }^{\dagger}$ by state ${ }^{\S} \_$United States, 2013 and $2017^{\mathfrak{T}}$

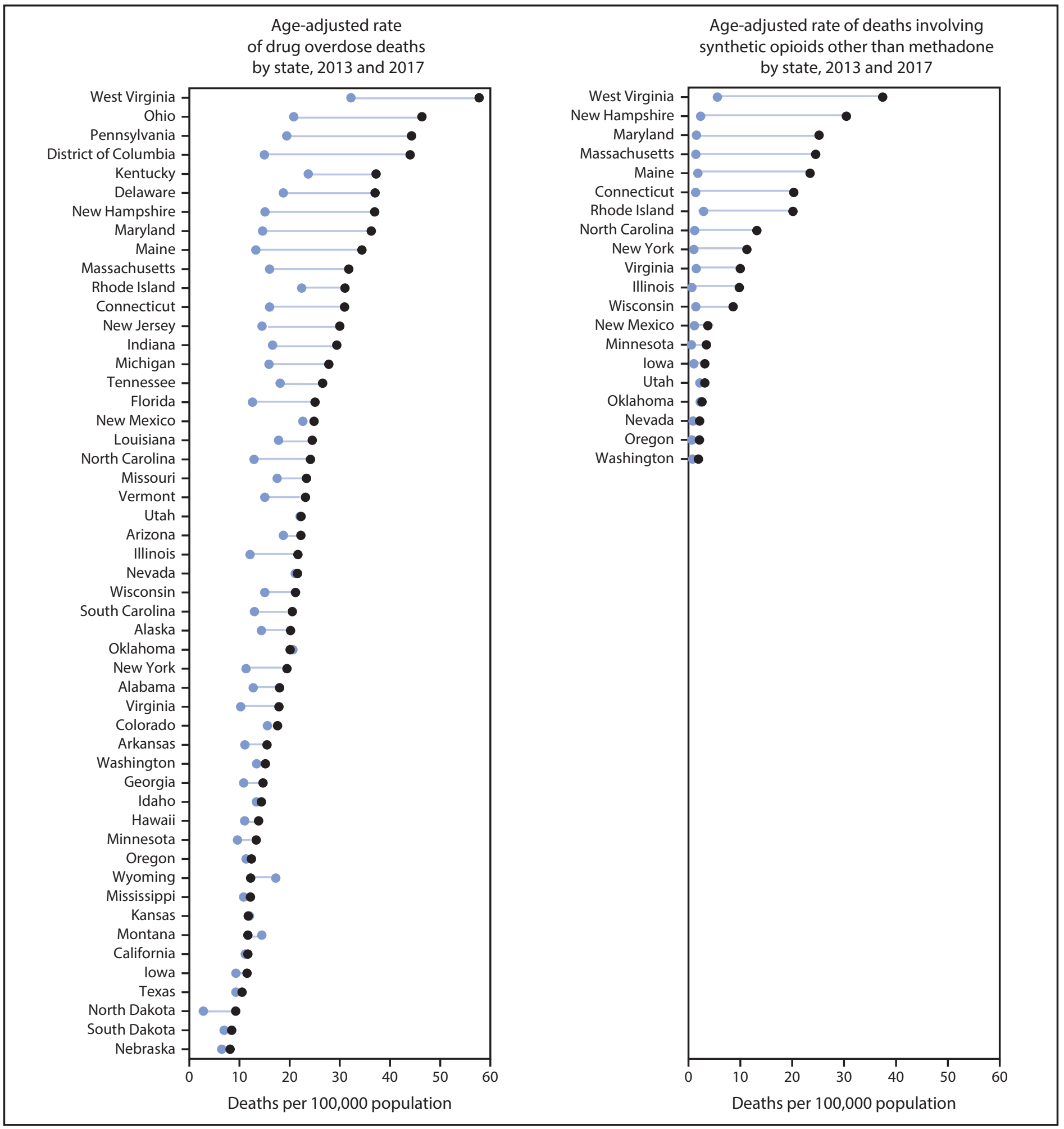

See footnotes on next page. 


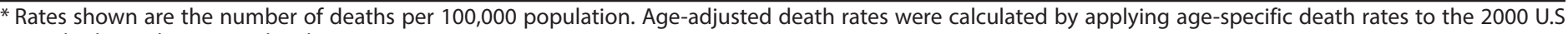
standard population age distribution.

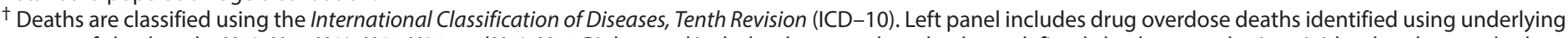

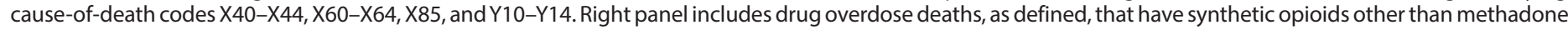
(T40.4) as a contributing cause.

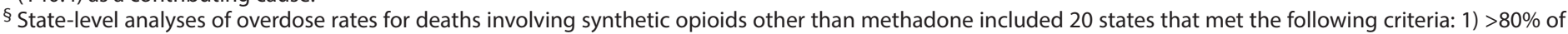

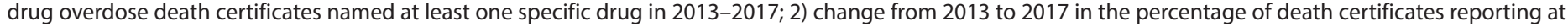

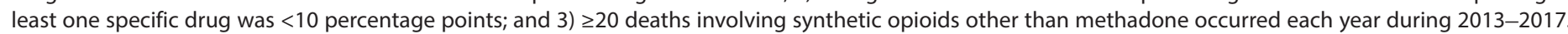

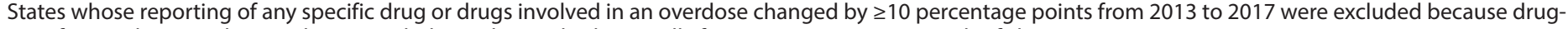
specific overdose numbers and rates might have changed substantially from 2013 to 2017 as a result of changes in reporting.

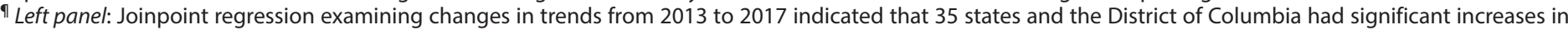

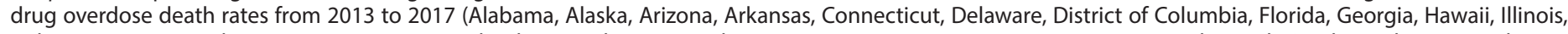

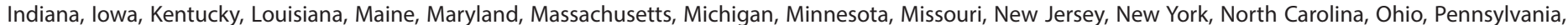

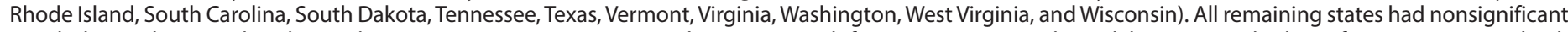

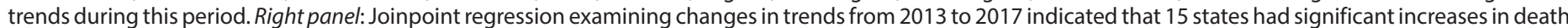

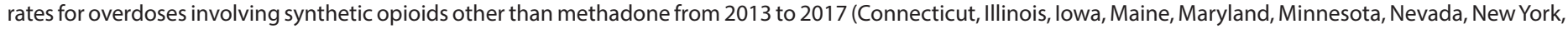

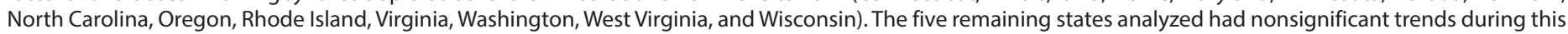

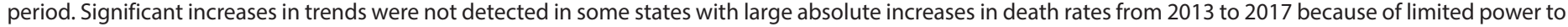
detect significant effects.
\end{abstract}

areas experienced the largest absolute rate increase (an increase of 1.9 per 100,000$)$, and the largest relative rate increase occurred in micropolitan counties (14.9\%). Death rates increased significantly in 15 states, with the largest relative changes in North Carolina (28.6\%), Ohio (19.1\%), and Maine (18.7\%).

From 2016 to 2017, the prescription opioid-involved death rate decreased $13.2 \%$ among males aged $15-24$ years but increased $10.5 \%$ among persons aged $\geq 65$ years (Table 1 ). These death rates remained stable from 2016 to 2017 across all racial groups and urbanization levels and in most states, although five states (Maine, Maryland, Oklahoma, Tennessee, and Washington) experienced significant decreases, and one (Illinois) had a significant increase. The largest relative changes included a $29.7 \%$ increase in Illinois and a 39.2\% decrease in Maine. The highest prescription opioid-involved death rates in 2017 were in West Virginia (17.2 per 100,000), Maryland (11.5), and Utah (10.8).

Heroin-involved overdose death rates declined among many groups in 2017 compared with those in 2016 (Table 2). The largest declines occurred among persons aged 15-24 years $(15.0 \%)$, particularly males $(17.5 \%)$, as well as in medium metro counties (6.1\%). Rates declined 3.2\% among whites. However, heroin-involved overdose death rates did increase among some groups; the largest relative rate increase occurred among persons aged $\geq 65$ years $(16.7 \%)$ and $55-64$ years $(11.6 \%)$ and among blacks (8.9\%). Rates remained stable in most states, with significant decreases in five states (Maryland, Massachusetts, Minnesota, Missouri, and Ohio), and increases in three (California, Illinois, and Virginia). The largest relative decrease $(31.9 \%)$ was in Ohio, and the largest relative increase $(21.8 \%)$ was in Virginia. The highest heroin-involved overdose death rates in 2017 were in DC (18.0 per 100,000), West Virginia (14.9), and Connecticut (12.4).
Deaths involving synthetic opioids propelled increases from 2016 to 2017 across all demographic categories (Table 2). The highest death rate was in males aged $25-44$ years $(27.0$ per $100,000)$, and the largest relative increases occurred among blacks (60.7\%) and American Indian/Alaska Natives (58.5\%). Deaths increased across all urbanization levels from 2016 to 2017. Twentythree states and DC experienced significant increases in synthetic opioid-involved overdose death rates, including eight states west of the Mississippi River. The largest relative rate increase occurred in Arizona (122.2\%), followed by North Carolina (112.9\%) and Oregon (90.9\%). The highest synthetic opioid-involved overdose death rates in 2017 were in West Virginia (37.4 per 100,000), Ohio (32.4), and New Hampshire (30.4).

\section{Discussion}

In the United States, drug overdoses resulted in 702,568 deaths during 1999-2017, with 399,230 (56.8\%) involving opioids. ${ }^{\dagger \dagger \dagger \dagger}$ From 2016 to 2017 , death rates from all opioids increased, with increases driven by synthetic opioids. Deaths involving IMF have been seen primarily east of the Mississippi River; ${ }^{\$ S \$}$ however, recent increases occurred in eight states west of the Mississippi River, including Arizona, California, Colorado, Minnesota, Missouri, Oregon, Texas, and Washington.

Drug overdose death rates from 2013 to 2017 increased in most states; the influence of synthetic opioids on these rate increases was seen in approximately one quarter of all states during this same 5-year period. Overdose deaths involving cocaine and psychostimulants also have increased in recent years $(1,6)$. Overall, the overdose epidemic continues to worsen, and it has grown increasingly complex by co-involvement of prescription and illicit drugs $(7,8) .9999$ For example, in 2016, synthetic opioids

††† https://wonder.cdc.gov

\$\$\$\$ https://emergency.cdc.gov/han/han00413.asp.

g999 https://www.cdc.gov/nchs/data/nvsr/nvsr67/nvsr67_09-508.pdf. 


\section{Summary}

What is already known about this topic?

The U.S. opioid overdose epidemic continues to evolve. In 2016, $66.4 \%$ of the 63,632 drug overdose deaths involved an opioid.

What is added by this report?

In 2017, among 70,237 drug overdose deaths, 47,600 (67.8\%) involved opioids, with increases across age groups, racial/ethnic groups, county urbanization levels, and in multiple states. From 2013 to 2017, synthetic opioids contributed to increases in drug overdose death rates in several states. From 2016 to 2017, synthetic opioid-involved overdose death rates increased $45.2 \%$.

What are the implications for public health practice?

Continued federal, state, and local surveillance efforts to inform evidence-based prevention, response, and treatment strategies and to strengthen public health and public safety partnerships are urgently needed.

(primarily IMF) were involved in $23.7 \%$ of deaths involving prescription opioids, $37.4 \%$ involving heroin, and $40.3 \%$ involving cocaine (9). In addition, death rates are increasing across multiple demographic groups. For example, although death rates involving opioids remained highest among whites, relatively large increases across several drug categories were observed among blacks.

The findings in this report are subject to at least five limitations. First, at autopsy, substances tested for vary by time and jurisdiction, and improvements in toxicologic testing might account for some reported increases. Second, the specific types of drugs involved were not included on $15 \%$ of drug overdose death certificates in 2016 and $12 \%$ in 2017, and the percentage of death certificates with at least one drug specified ranged among states from $54.7 \%-99.3 \%$ in 2017 , limiting rate comparisons between states. Third, because heroin and morphine are metabolized similarly (10), some heroin deaths might have been misclassified as morphine deaths, resulting in underreporting of heroin deaths. Fourth, potential race misclassification might have led to underestimates for certain categories, primarily for American
Indian/Alaska Natives and Asian/Pacific Islanders. ${ }^{* * * * *}$ Finally, most state-specific analyses were restricted to DC and a subset of states with adequate drug specificity, limiting generalizability.

Through 2017, the drug overdose epidemic continues to worsen and evolve, and the involvement of many types of drugs (e.g., opioids, cocaine, and methamphetamine) underscores the urgency to obtain more timely and local data to inform public health and public safety action. Although prescription opioidand heroin-involved death rates were stable from 2016 to 2017 , they remained high. Some preliminary indicators in 2018 point to possible improvements based on provisional data; ${ }^{\dagger+t^{\dagger \dagger}}$ however, confirmation will depend on results of pending medical investigations and analysis of final data. Overall, deaths involving synthetic opioids continue to drive increases in overdose deaths. CDC funds 32 states and DC to collect more timely and comprehensive drug overdose data, including improved toxicologic testing in opioid-involved fatal overdoses. $\$ \$ S \$ S \$$ CDC is funding prevention activities in 42 states and DC.99999 $\mathrm{CDC}$ also is leveraging emergency funding to support 49 states, DC, and four territories to broaden their surveillance and response capabilities and enable comprehensive communitylevel responses with implementation of novel, evidence-based interventions. $^{* * * * * *}$ Continued efforts to ensure safe prescribing practices by following the CDC Guideline for Prescribing Opioids for Chronic Pain ${ }^{\dagger \dagger \dagger \dagger \dagger}$ are enhanced by access to nonopioid and nonpharmacologic treatments for pain. Other important activities include increasing naloxone availability, expanding access to medication-assisted treatment, enhancing public health and public safety partnerships, and maximizing the ability of health systems to link persons to treatment and harm-reduction services.

\footnotetext{
***** https://www.cdc.gov/nchs/data/series/sr_02/sr02_172.pdf.

计柿 https://www.cdc.gov/nchs/nvss/vsrr/drug-overdose-data.htm.

$\$ \$ S \$ S$ https://www.cdc.gov/drugoverdose/foa/state-opioid-mm.html.

9999 https://www.cdc.gov/drugoverdose/states/state_prevention.html. https://www. cdc.gov/drugoverdose/foa/ddpi.html.

*a*k***https://www.cdc.gov/cpr/readiness/funding-opioid.htm.

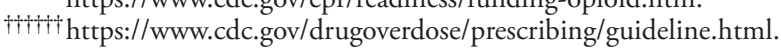




\section{Acknowledgment}

Rose Rudd, MSPH, Division of Unintentional Injury Prevention, National Center for Injury Prevention and Control, CDC.

Corresponding authors: Lawrence Scholl, 1zi8@cdc.gov, 404-498-1489; Puja Seth, pseth@cdc.gov, 404-639-6334.

${ }^{1}$ Division of Unintentional Injury Prevention, National Center for Injury Prevention and Control, CDC.

All authors have completed and submitted the ICMJE form for disclosure of potential conflicts of interest. No potential conflicts of interest were disclosed.

\section{References}

1. Seth P, Scholl L, Rudd RA, Bacon S. Overdose deaths involving opioids, cocaine, and psychostimulants-United States, 2015-2016. MMWR Morb Mortal Wkly Rep 2018;67:349-58. https://doi.org/10.15585/ mmwr.mm6712a1

2. Gladden RM, Martinez P, Seth P. Fentanyl law enforcement submissions and increases in synthetic opioid-involved overdose deaths - 27 states, 2013-2014. MMWR Morb Mortal Wkly Rep 2016;65:837-43. https:// doi.org/10.15585/mmwr.mm6533a2

3. O'Donnell JK, Gladden RM, Seth P. Trends in deaths involving heroin and synthetic opioids excluding methadone, and law enforcement drug product reports, by census region-United States, 2006-2015. MMWR Morb Mortal Wkly Rep 2017;66:897-903 10.15585/mmwr. mm6634a2. https://doi.org/10.15585/mmwr.mm6634a2
4. O’Donnell JK, Halpin J, Mattson CL, Goldberger BA, Gladden RM. Deaths involving fentanyl, fentanyl analogs, and U-47700 - 10 states, JulyDecember 2016. MMWR Morb Mortal Wkly Rep 2017;66:1197-202. https://doi.org/10.15585/mmwr.mm6643e1

5. Seth P, Rudd RA, Noonan RK, Haegerich TM. Quantifying the epidemic of prescription opioid overdose deaths. Am J Public Health 2018;108:500-2. https://doi.org/10.2105/AJPH.2017.304265

6. McCall Jones C, Baldwin GT, Compton WM. Recent increases in cocaine-related overdose deaths and the role of opioids. Am J Public Health 2017;107:430-2. https://doi.org/10.2105/AJPH.2016.303627

7. Kandel DB, Hu MC, Griesler P, Wall M. Increases from 2002 to 2015 in prescription opioid overdose deaths in combination with other substances. Drug Alcohol Depend 2017;178:501-11. https://doi. org/10.1016/j.drugalcdep.2017.05.047

8. Mattson CL, O’Donnell J, Kariisa M, Seth P, Scholl L, Gladden RM. Opportunities to prevent overdose deaths involving prescription and illicit opioids, 11 states, July 2016-June 2017. MMWR Morb Mortal Wkly Rep 2018;67:945-51. https://doi.org/10.15585/mmwr. mm6734a2

9. Jones CM, Einstein EB, Compton WM. Changes in synthetic opioid involvement in drug overdose deaths in the United States, 2010-2016. JAMA 2018;319:1819-21. https://doi.org/10.1001/jama.2018.2844

10. Davis GG; National Association of Medical Examiners and American College of Medical Toxicology Expert Panel on Evaluating and Reporting Opioid Deaths. Complete republication: National Association of Medical Examiners position paper: recommendations for the investigation, diagnosis, and certification of deaths related to opioid drugs. J Med Toxicol 2014;10:100-6. https://doi.org/10.1007/s13181-013-0323-x 